\title{
Static Gravitational Global Monopoles
}

\author{
Steven L. Liebling \\ Theoretical and Computational Studies Group \\ Southampton College, Long Island University, Southampton, NY 11968
}

\begin{abstract}
Static solutions in spherical symmetry are found for gravitating global monopoles. Regular solutions lacking a horizon are found for $\eta<1 / \sqrt{8 \pi}$, where $\eta$ is the scale of symmetry breaking. Apparently regular solutions with a horizon are found for $1 / \sqrt{8 \pi} \leq \eta \lesssim \sqrt{3 / 8 \pi}$. Though they have a horizon, they are not Schwarzschild. The solution for $\eta=1 / \sqrt{8 \pi}$ is argued to have a horizon at infinity. The failure to find static solutions for $\eta>\sqrt{3 / 8 \pi} \approx 0.3455$ is consistent with findings that topological inflation begins at $\eta \approx 0.33$.
\end{abstract}

04.25.Dm, 04.70.Bw, 04.40.-b

Topological defects have attracted quite a bit of attention because of their relevance to a number of different areas ranging from condensed matter to structure formation. Studies of global monopoles in particular have served as a foundation on which knowledge of other defects has been built. Previous work details static gravitating global monopole solutions [1,2], while further studies consider the gauged case 3 - 5n. Understanding of the static solutions is also relevant to the study of topological inflation [6,8].

Here I return to the global monopole case and consider the possibility that static global monopoles have a horizon (the "rather curious" monopoles mentioned in [2]). I reproduce the solutions of [1] for $\eta<1 / \sqrt{8 \pi}$, and find other solutions for $1 / \sqrt{8 \pi} \leq \eta \lesssim \sqrt{3 / 8 \pi}$ which, though they contain a horizon, appear regular. I also comment on the possibility that the failure to find static solutions for $\eta>\sqrt{3 / 8 \pi}$ is indicative of the onset of topological inflation which has been reported for $\eta \gtrsim 0.33$ [8 10].

Letting $\Phi^{a}$ represent a triplet scalar field and including the usual symmetry breaking potential with scale of symmetry breaking $\eta$, the Lagrangian is

$$
L=-\frac{1}{2} \Phi^{a ; \mu} \Phi_{; \mu}^{a}-\frac{1}{4} \lambda\left[\left(\Phi^{a}\right)^{2}-\eta^{2}\right]^{2}
$$

where $\lambda$ is a coupling constant which sets the scale. Henceforth, I choose $\lambda=0.1$ without loss of generality.

The spherically symmetric metric

$$
d s^{2}=-A^{2} \mu d t^{2}+\frac{1}{\mu} d r^{2}+r^{2} d \Omega^{2}
$$

is adopted in terms of the metric components $A(r)$ and $\mu(r)$ (the same as in [3] modulo the sign convention). By association with the Schwarzschild metric, a mass aspect function $m(r)$ is defined

$$
m(r) \equiv \frac{r}{2}(1-\mu) .
$$

The usual hedgehog ansatz for the triplet field, $\Phi^{a}=$ $f(r) \hat{r}$, is chosen in terms of the monopole profile $f(r)$.
Casting the equations in first-order form, an auxiliary variable $\Psi(r) \equiv f^{\prime}$ is introduced (a prime denotes $d / d r$ ). The equations for static solutions then become

$$
\begin{aligned}
f^{\prime} & =\Psi \\
\Psi^{\prime} & =\frac{f}{r^{2} \mu}\left[2+\lambda r^{2}\left(f^{2}-\eta^{2}\right)\right]-\Psi\left(\frac{2}{r}+4 \pi r \Psi^{2}+\frac{\mu^{\prime}}{\mu}\right) \\
\mu^{\prime} & =\frac{1-\mu}{r}-4 \pi r \Psi^{2} \mu-8 \pi\left[\frac{f^{2}}{r}+\frac{\lambda r}{4}\left(f^{2}-\eta^{2}\right)^{2}\right] \\
A^{\prime} & =4 \pi r A \Psi^{2} .
\end{aligned}
$$

Imposing regularity as $r \rightarrow \infty$, these equations show that $\mu^{\prime} \rightarrow 0$ and $\mu \rightarrow 1-8 \pi \eta^{2}$. Confirmation that the numerical solutions obey this behavior is shown in Fig. 1. Thus, the space has a deficit solid angle $\Delta=4 \pi\left(8 \pi \eta^{2}\right)$ [8].

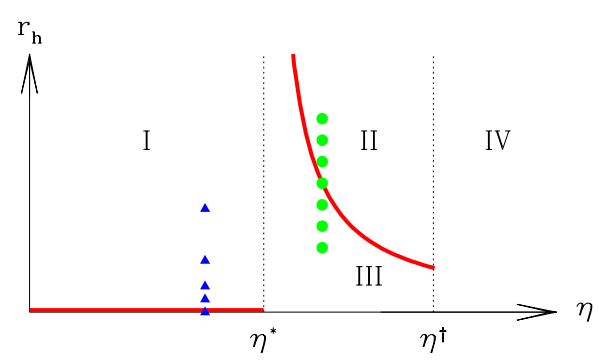

FIG. 1. Schematic diagram of the solution space of static solutions. Regular solutions are represented by the two disjoint bold curves. The radius of any horizon $r_{h}$ is plotted versus $\eta$. The regions denote the types of singular solutions: (I) black hole solutions, (II) a single horizon, (III) two horizons, (IV) no static solutions found. The family of solutions denoted by solid triangles is shown in Fig. 2. The family denoted by solid circles is shown in Fig. 3 .

These equations have singularities at $r=0$ and where $\mu=0$. To integrate outward from $r=0$, regularity is assumed, and by Taylor expanding about $r=0$, the solutions can be integrated from close to the origin. Specifically, the conditions 


$$
\begin{array}{ll}
\mu(0)=1 & \mu^{\prime}(0)=0 \\
f(0)=0 & \Psi^{\prime}(0)=0
\end{array}
$$

apply, and $\Psi(0)$ is a free parameter which is adjusted via a standard shooting method until the correct asymptotic behavior for $f(r)$ is observed, specifically $f(r \rightarrow \infty)=\eta$.

Solutions where $\mu(r)$ vanishes can be handled in a similar fashion. Defining $r_{h}$ to be the radius of the horizon such that $\mu\left(r_{h}\right) \equiv 0$, appropriate boundary conditions at $r=r_{h}$ can be found by enforcing regularity there. Then by Taylor expanding about $r_{h}$, the solutions can be integrated either outward or inward from near the horizon. The value $f\left(r_{h}\right)$ is then a free parameter which is adjusted via a shooting method so that, if integrating outward, the solution satisfies $f(r \rightarrow \infty)=\eta$, or, if inward, satisfies $f(r \rightarrow 0)=0$. A standard ODE integrator has been used.

The solutions found are summarized in a schematic of the solution space in Fig. 1. Regular solutions lacking a horizon are found as $\eta$ is increased to a critical value, $\eta^{*} \equiv 1 / \sqrt{8 \pi} \approx 0.1995$. For $\eta \geq \eta^{*}$, static solutions with an apparently regular horizon are found up to $\eta \approx$ 0.3455. Empirically it appears that this upper limit for $\eta$ occurs at $\sqrt{3 / 8 \pi}$, although unlike the case of $\eta^{*}$ no theoretical justification for this limit is found. Above this second critical value $\eta^{\dagger} \equiv \sqrt{3 / 8 \pi}$, no static solutions are found. In addition to the regular static solutions, singular solutions are also found as discussed below.

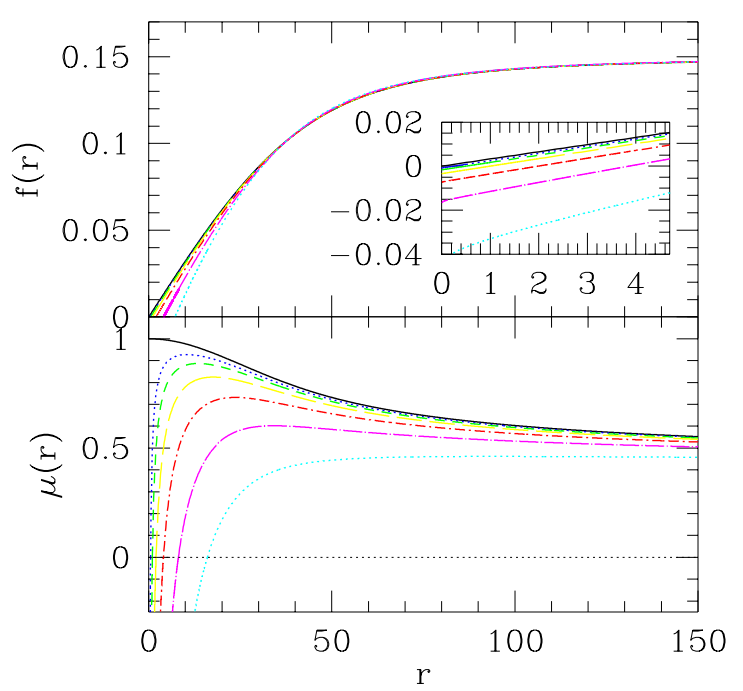

FIG. 2. Family of static solutions for $\eta=0.15$. One solution (solid) is found to be regular and static, while the other solutions contain a horizon at $r_{h}=0.5,1,2,4,8,16$ and are singular at the origin. Where $\mu(r)$ vanishes denotes a horizon.

For $\eta<1 / \sqrt{8 \pi}$, horizonless static solutions exist as described in [1]. A typical example of such a solution is shown in Fig. 2. Other solutions are also shown which are singular at $r=0$ and correspond to black holes containing a monopole charge. These singular solutions are obtained by enforcing the existence of a horizon at a particular radius and demanding that $f(r \rightarrow \infty)=\eta$. For a given value of $\eta$, a family of static solutions exists, only one member of which is regular.

Fig. 2 shows that asymptotically the solutions approach one another independent of the existence of the black hole. Such a convergence of the singular solutions to the regular solution for $r_{h} \rightarrow 0$ is also observed for the gauged monopole in [3]. Furthermore, in contrast to the solution for a gauged monopole, the metric component $\mu$ does not asymptote to unity as it would in an asymptotically flat spacetime. Instead, that it asymptotes to a non-unit value indicates the linear divergence of the mass of an isolated global monopole.

Solutions similar to those shown in Fig. 2 are found for $\eta$ increasing until $\eta \approx 0.20 \approx \eta^{*}$. As $\eta$ is increased, the asymptotic value of $\mu$ decreases toward zero. For $\eta \gtrsim 0.20$, no solutions without $\mu$ vanishing are found. This result agrees with the argument presented in [8] that when $\eta>1 / \sqrt{8 \pi}$ no static (horizonless) solutions exist.

For the critical case in which $\eta=\eta^{*}$, the solution has $\mu$ vanishing at $r=\infty$. This solution then represents a static spacetime with a horizon at infinity. It is not clear if there are any cosmological implications to the existence of such a solution.

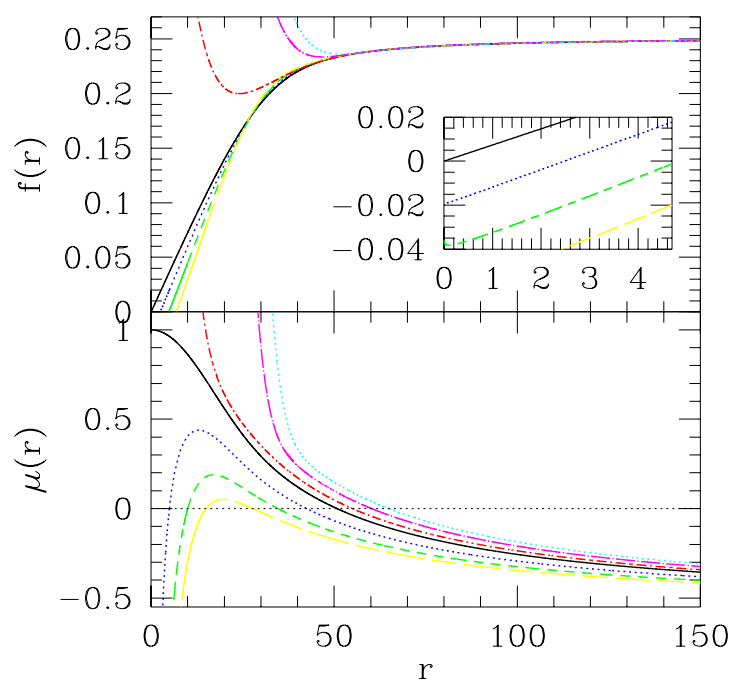

FIG. 3. Family of static solutions for $\eta=0.25$. One solution (solid) is found to be regular and has a horizon at $r_{h}=50.78$. Three solutions singular at the origin are shown containing two horizons, the first occurring at $r_{h}=5,10,15$. Three other singular solutions having only one horizon are shown with $r_{h}=55,60,65$. Compare to the sub-critical case shown in Fig. 目.

For $\eta>\eta^{*}$, static solutions can be found, albeit with a 
horizon. One such example is displayed in Fig. 3. Shooting from the origin, the radius at which $\mu$ vanishes, $r_{h}$, can be determined. Then, by Taylor expanding about $r_{h}$, the solution can be extended to large $r$. The solution is thus regular both at $r=0$ and at $r=r_{h}$. Once again, irregular solutions can be constructed by enforcing the vanishing of $\mu$ at some other radius. Solutions with horizons smaller than that of the regular solution have two horizons as shown in the figure, while those with horizon greater than that of the regular solution have only one.

I note that none of these solutions represents a black hole as $\mu$ is negative for large $r$ and positive for small $r$, the opposite of Schwarzschild. As is evident from the metric (2), the roles of $t$ and $r$ switch outside the vanishing of $\mu$. Hence, the exterior is no longer static though the solution remains independent of $t$. However, within the horizon these solutions remain static.

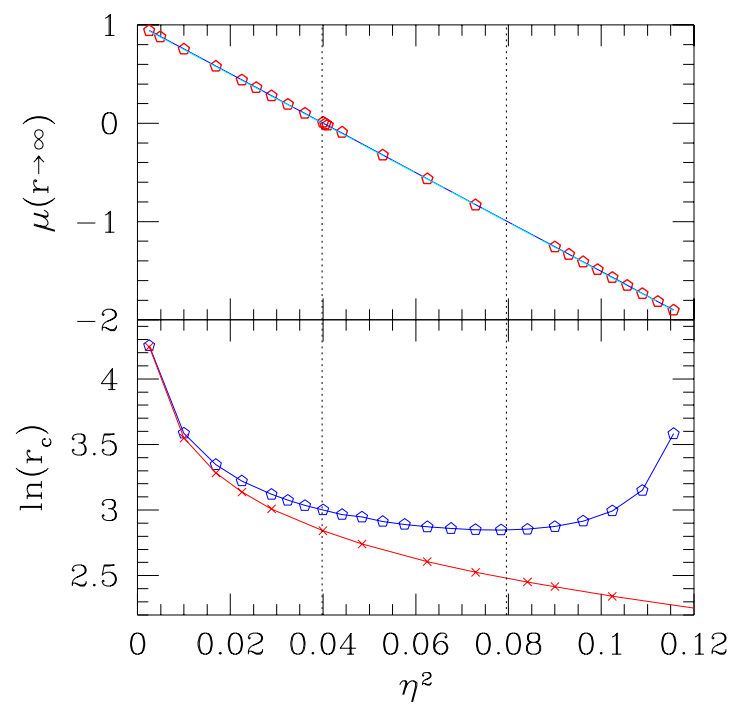

FIG. 4. The top frame shows the asymptotic behavior of $\mu(r)$. The points represent the asymptotic value of $\mu$ computed via the slope of a least-squares fit to $r \mu$ versus $r$ for each $\eta$. The solid line shows the least-squares fit to the points, while the dashed line (indistinguishable from the fit) shows the expected relationship $\mu(r \rightarrow \infty)=1-8 \pi \eta^{2}$. The vertical dotted lines denote $\eta^{*}$ and $\eta^{\natural}$. The bottom frame shows the core radius (open pentagons) which reaches a minimum at $\left(\eta^{\natural}\right)^{2} \approx 0.08$. For comparison, the core radius for flat-space monopoles (cross hatches) is also shown.

As $\eta$ is increased, another transition is evident near $\eta \approx 0.28$. This transition occurs at a new critical value of $\eta$, namely $\eta^{\natural} \equiv \sqrt{2 / 8 \pi}$, and is observed by examining the size of the monopole. Defining the core radius by $f\left(r_{c}\right) \equiv \eta / 2$, I show $r_{c}$ versus $\eta$ in Fig. 月. For $\eta<\eta^{\natural}$, the core radius decreases with increasing $\eta$, while for $\eta>\eta^{\natural}$ the radius decreases. Oscillations in the solution also become evident for $\eta>\eta^{\natural}$ as shown in Fig. 6.

These oscillatory solutions bear a striking resemblance to the reported stable solutions found for the dynamical evolutions of gauged monopoles with large $\eta$ in [9]. The oscillations become more pronounced as $\eta$ approaches $\eta^{\dagger}$, and is accompanied by a dramatic decrease in the minimum to which $\mu$ reaches. For $\eta>\eta^{\dagger} \approx 0.3455$ no static solutions are found.

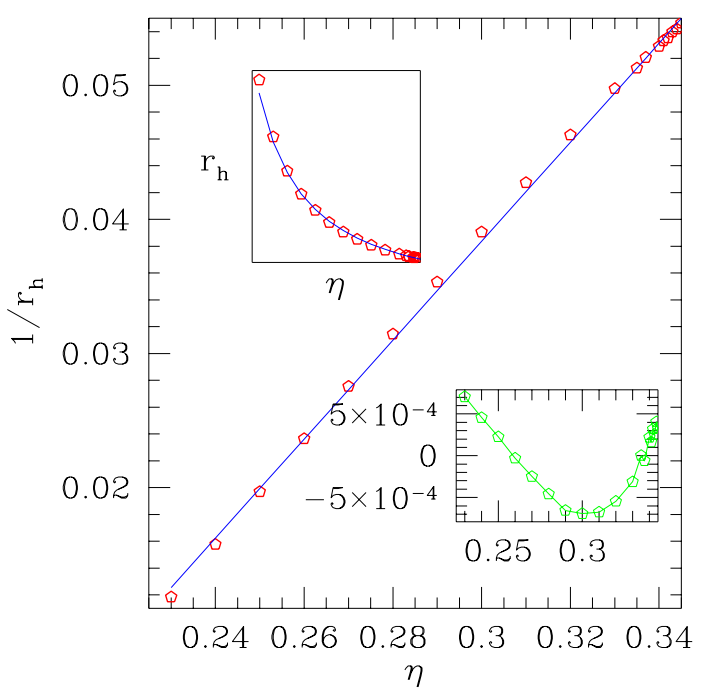

FIG. 5. Behavior of the horizon radius $r_{h}$ of the static super-critical solutions versus $\eta$. The solid line indicates a least-squares fit of $1 / r_{h}=A \eta+B$ where $A=0.3690$ and $B=-0.0723$. The upper insert shows the same data where $r_{h} \propto 1 /(\eta-C)$ where $C=-B / A=0.1959$. It is expected that this value of $C$ would be $\eta^{*} \approx 0.1995$. The lower inset displays the deviation from the fit.

Interestingly for super-critical solutions, the horizon $r_{h}$ of the regular solutions obeys a scaling law in $\eta$. In particular, the horizon radius is found to obey

$$
r_{h} \propto \frac{1}{\eta-\eta^{*}}
$$

as demonstrated in Fig. 5 .

A summary of the solutions regular at the origin are shown in Fig. 6. As $\eta$ is increased, the asymptotic value of $\mu$ is seen to decrease below zero indicating the presence of a horizon. As $\eta$ is increased further, the value of $\mu$ continues to decrease until $\eta=\eta^{\dagger} \approx 0.3455$ above which no static solutions are found.

The three critical values of $\eta$ all correspond to integer multiples of a deficit solid angle of $4 \pi$. The deficit solid angle $\Delta^{*}$ occurring for $\eta=\eta^{*}$ is known to be precisely $\Delta^{*} \equiv 4 \pi$. This critical value denotes the transition to static solutions with horizons. The next transition occurs when the monopole core radius changes from decreasing to increasing with $\eta$, namely $\Delta^{\natural} \equiv 2 \Delta^{*}$. Finally, the transition above which no static solutions are found occurs at $\Delta^{\dagger} \equiv 3 \Delta^{*}$. These latter two transitions are found only empirically, calling for a geometric explanation. 


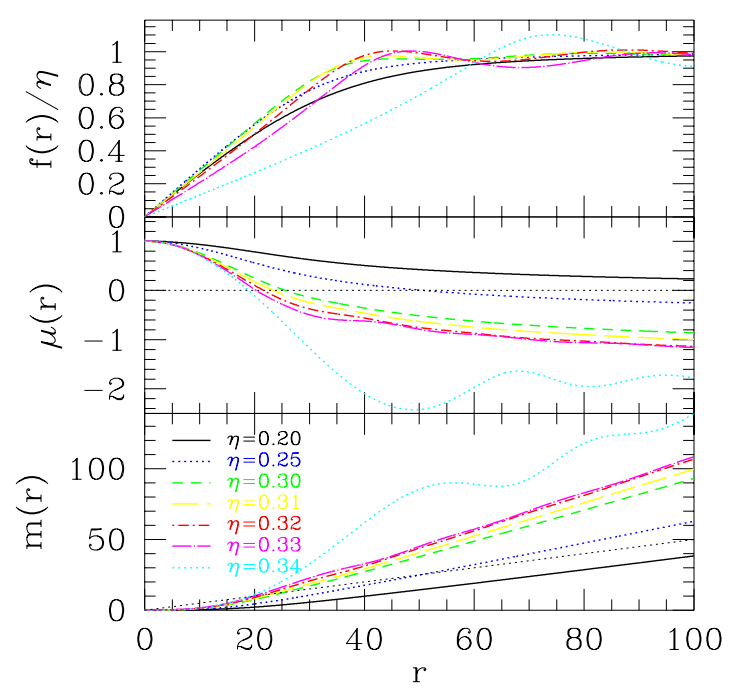

FIG. 6. Set of static, regular solutions for $\eta \in[0.20,0.34]$. The top frame shows the rescaled hedgehog profile. The middle frame shows the metric component $\mu$ with horizons indicated by $\mu=0$ (the dotted line). The bottom frame shows the mass aspect function with horizons indicated by $m=r / 2$ (the dotted line). Note that the core radius decreases and then increases as $\eta$ increases, the transition occurring for $\eta \approx 0.28$.

Having the static solutions in hand, the next question to consider is whether they are stable. In particular, a relevant question is whether the sub-critical static solutions are unstable to collapse to a black hole. Ortiz asks this question of the gauged monopoles, and answers it by considering the mass of the various solutions [4]. Where the monopole has greater mass than a black hole with the same topological charge, the solution would be expected to be unstable.

However, the mass of the global monopole is divergent, and so it is not clear if such an argument can be made here. The quantity $2 m / r$, equal to $1-\mu$, does asymptote to a finite value. Perhaps comparing the mass within a particular radius would be sufficient to answer the question. Fig. 2 2 shows, for the sub-critical case, the behavior of $\mu$ for both the regular solution and various black hole solutions. The black hole solutions do have more mass (smaller $\mu$ ) than the regular solution for finite radius (i.e. "locally"), however they asymptote to the same value at infinity. Independent of whether one looks at the local or asymptotic value of $2 m / r$, the regular solutions do not have greater mass than the black hole, and this fact might be some indication that the solutions are indeed stable. Evolutions conducted in [11] also indicate that the solutions are stable.

Considering now the super-critical case, are these static solutions unstable to some other solution? From Fig. 3, one could consider the stability of the regular solution to the irregular ones having either one or two hori- zons. However, the physical significance of those horizons is not clear.

Instead, it is more interesting to examine these results in the context of topological inflation [6 10]. As reported in [8, 10], when $\eta \gtrsim 0.33$ the region inside a global monopole necessarily undergoes inflation. The square of this value falls roughly in the middle of the two transitions $\left(\eta^{\natural}\right)^{2}$ and $\left(\eta^{\dagger}\right)^{2}$, and thus it appears that topological inflation does not begin at either of the transitions.

Instead, a change in stability appears likely near $\eta \approx$ 0.33. A linear perturbation analysis should be able to confirm both the change in stability and the critical value of $\eta$ for which topological inflation begins.

Acknowledgments: I am grateful for helpful discussions with Arvind Borde, Inyong Cho, Eric Hirschmann, Nobuyuki Sakai, and Alexander Vilenkin. I am also thankful for the support of the Southampton College Research \& Awards Committee.

[1] M. Barriola and A. Vilenkin, "Gravitational Field Of A Global Monopole," Phys. Rev. Lett. 63, 341 (1989).

[2] D. Harari and C. Loustó, "Repulsive Gravitational Effects Of Global Monopoles," Phys. Rev. D42, 2626 (1990).

[3] P. Breitenlohner, P. Forgacs and D. Maison, "Gravitating monopole solutions," Nucl. Phys. B383, 357 (1992).

[4] M.E. Ortiz, "Curved space magnetic monopoles," Phys. Rev. D45, 2586 (1992).

[5] K. Lee, V.P. Nair and E.J. Weinberg, "Black holes in magnetic monopoles," Phys. Rev. D45, 2751 (1992) hepth/9112008

[6] A. Vilenkin, "Topological inflation," Phys. Rev. Lett. 72, 3137 (1994) hep-th/9402085.

[7] A. Linde, "Monopoles as big as a universe," Phys. Lett. B327, 208 (1994) astro-ph/9402031.

[8] I. Cho and A. Vilenkin, "Space-time structure of an inflating global monopole," Phys. Rev. D56, 7621 (1997) gr-qc/9708005.

[9] N. Sakai, "Dynamics of gravitating magnetic monopoles," Phys. Rev. D54, 1548 (1996) gr-qc/9512045.

[10] N. Sakai, H. Shinkai, T. Tachizawa, K. Maeda, "Dynamics of topological defects and inflation," Phys. Rev. D53, 655 (1996). gr-qc/9506068.

[11] S.L. Liebling, "Critical phenomena inside global monopoles," gr-qc/9904077. 